\section{SURVIVAL OUTCOME AND RECURRENCE PATTERN AFTER LYMPHADENECTOMY FOR ENDOMETRIAL CANCER}

'AG Nath*, 'S Sambasivan, ${ }^{2} S \mathrm{~J},{ }^{3} \mathrm{~J}$ Krishna, 'PN Rema. 'rec, gynaecological oncology, Trivandrum, India; ${ }^{2} r c c$, surgical oncology, Trivandrum, India; ${ }^{3} r c c$, biostatistics, Trivandrum, India

\subsection{6/ijgc-2021-ESGO.172}

Introduction/Background* Standard surgical treatment for carcinoma endometrium comprise hysterectomy with pelvic and para aortic node dissection followed by adjuvant treatment depending on final pathology report. Systematic lymphadenectomy in endometrial cancer still remains as a controversial topic.

Methodology To assess pattern of lymph node metastasis, pelvic control rate and overall and disease free survival outcome of cancer endometrium patients who underwent lymphadenectomy along with hysterectomy.

Retrospective audit of patients who underwent lymphadenectomy as a part of staging from January 2010-December 2016. It includes 156 patients. Survival curves plotted using Kaplan-Meier method. Statistical significance assessed using log-rank test.

Result(s)* Median follow up was 60 months. Overall survival (OS) and disease free survival (DFS) was significantly better in patients who underwent systematic pelvic and paraaortic node dissection rather than pelvic node dissection alone for stage III and IV Carcinoma endometrium $(p=0.025)$. Multivariate analysis showed that lymph node dissection, age, lymph node metastasis, and adjuvant therapy were independent prognostic variables.

Conclusion* Systematic pelvic and paraaortic lymph node dissection significantly prolongs the survival of patients with stage III and IV as well as grade 3 and type II carcinoma endometrium. Most common site of tumour recurrence in patients who didn't undergo para aortic lymphadenectomy was para aortic area.

\section{A VALIDATION STUDY OF MODIFIED TCGA CLASSIFICATION FOR PATIENTS WITH ENDOMETRIAL CANCER TREATED WITH RADICAL SURGERY AND ADJUVANT CHEMOTHERAPY}

${ }^{1} \mathrm{H}$ Yamazaki*, ${ }^{1} \mathrm{H}$ Asano, ${ }^{2} \mathrm{~K}$ Hatanaka, ${ }^{3} \mathrm{R}$ Matsuoka, ${ }^{2} \mathrm{~K}$ Ihira, ${ }^{2} \mathrm{D}$ Endo, ${ }^{2} \mathrm{~T}$ Mitamura, ${ }^{2} Y$ Konno, ${ }^{2} \mathrm{~T}$ Kato, ${ }^{2} \mathrm{Y}$ Hatanaka, ${ }^{1} \mathrm{H}$ Watari. ${ }^{1}$ Hokkaido University, Sapporo, Japan; ${ }^{2}$ Hokkaido University Hospital, Sapporo, Japan; ${ }^{3}$ International University of Health and Welfare, Otawara, Japan

\subsection{6/ijgc-2021-ESG0.173}

Introduction/Background* TCGA molecular classification for endometrial cancer is expected to propose the most appropriate treatment strategy for each patient. Most of the patients in previous studies on this issue from western countries were treated with adjuvant radiation therapy. Thus, the prognostic stratifications by TCGA molecular classification remain unclear when adjuvant chemotherapy is applied. In this study, we aimed to validate a modified TCGA classification.

Methodology From 2003 to 2015, the patients with endometrial cancer treated in our hospital were enrolled in this retrospective study. The patients who were surgically staged by hysterectomy, bilateral salpingo-oophorectomy, and systematic lymphadenectomy were included, and postoperative adjuvant chemotherapy was applied for the patients at intermediate or high-risk of recurrence. The patients with follow-up period of less than 3 years were excluded. The patients were molecularly classified in combination with conventional recurrent low-risk, and immunohistochemistry (IHC) for PMS2, MSH6 and p53, resulting in four subgroups: low-risk for recurrence by risk classification (Stage IA, Endometrioid carcinoma, Grade 1or2, negative lymphvascular space invasion), MMRd (PMS2 and/or MSH6 negative), p53abn (p53 positive), and NSMP (none of the above). The 5-year disease-specific survival rates (5y-DSS) were compared among each group.

Result(s)* Total of 175 patients were analyzed. Of 175 patients, 41 patients were classified as low risk for recurrence, 45 patients as MMRd, 19 patients as p53abn, and the remaining 45 patients as NSMP. The median age (58 years old) and follow-up period (104 months) were not significantly different among 4 groups. The distribution of FIGO stage or histological types were also not significantly different except for lowrisk group. The 5y-DSS was 100\%, 93\%, 74\%, and 91\%, respectively, and the prognosis was stratified into 3 groups: favorable, low-risk for recurrence; intermediate, MMRd and NSMP; unfavorable, p53abn.

Conclusion* We conclude that the prognosis can be stratified by a modified TCGA classification in combination with conventional recurrent risk and IHC for PMS2, MSH6 and p53 in surgically-staged endometrial cancer receiving adjuvant chemotherapy.

\section{DIAGNOSTIC ACCURACY OF PET/CT IN THE DETECTION OF RETROPERITONEAL LYMPH NODE IN PATIENTS WITH HIGH-RISK ENDOMETRIAL CANCER: META-ANALYSIS}

${ }^{1} \mathrm{~N}$ Manzour*, ${ }^{1} \mathrm{E}$ Chacon, ${ }^{1} \mathrm{~F}$ Boria, ${ }^{2} \mathrm{~A}$ Bronte, ${ }^{3} \mathrm{D}$ Sanin, ${ }^{4} \mathrm{~N}$ Martín-Calvo, ${ }^{1} \mathrm{JA}$ Mínquez, ${ }^{1} \mathrm{D}$ Vazquez, ${ }^{1} \mathrm{~T}$ Castellano, ${ }^{5} \mathrm{D}$ Salas, ${ }^{6} \mathrm{M}$ Gimeno, ${ }^{1} \mathrm{I}$ Brotons, ${ }^{1} \mathrm{~J}$ Vara, ${ }^{5} \mathrm{JM}$ Aramendia, ${ }^{6} \mathrm{M}$ Cambeiro, ${ }^{5} \mathrm{~L}$ Sánchez Lorenzo, ${ }^{5} \mathrm{~A}$ Gonzalez-Martin, ${ }^{2} \mathrm{MJ}$ Garcia Velloso, ${ }^{1} \mathrm{LM}$ Chiva, ${ }^{1} \mathrm{JL}$ Alcazar. ${ }^{1}$ Clinica Universidad de Navarra, Gynecology; ${ }^{2}$ Clinica universidad de Navarra, Medicina Nuclear, Pamplona, Spain; ${ }^{3}$ Clinica El Rosario, Gynecology, Colombia; ${ }^{4}$ Clinica Universidad de Navarra; ${ }^{5}$ Clinica Universidad de Navarra, Medical Oncology; ${ }^{6}$ Clinica Universidad de Navarra, Radiotherapy

\subsection{6/ijgc-2021-ESGO.174}

Introduction/Background* The aim of this study was to assess the diagnostic accuracy of preoperative ${ }^{18}$ F-FDG PET or PET/ CT in detecting retroperitoneal lymph node (pelvic lymph node and para-aortic lymph node) metastasis in patients with high-risk endometrial cancer.

Methodology A systematic literature review was performed in PubMed and Web of science with the terms 'endometrial cancer' and 'positron emission tomography. Inclusion criteria were prospective or retrospective studies that evaluated lymph node metastases in the retroperitoneal area in high-risk endometrial carcinoma and provided the necessary information to construct the $2 \times 2$ table. For the quantitative analysis, the pool sensitivity and the pool specificity for node detection was calculated. The quality of the studies was assessed with the QUADAS-2.

Result(s)* The research resulted in 478 eligible citations from January 1990 to May 2020. After the exclusion, fourteen articles that met all the inclusion criteria were included, comprising data from 850 patients. The sensitivity, specificity and AUC of 18 F-FDG PET or PET/CT in the detection of lymph 


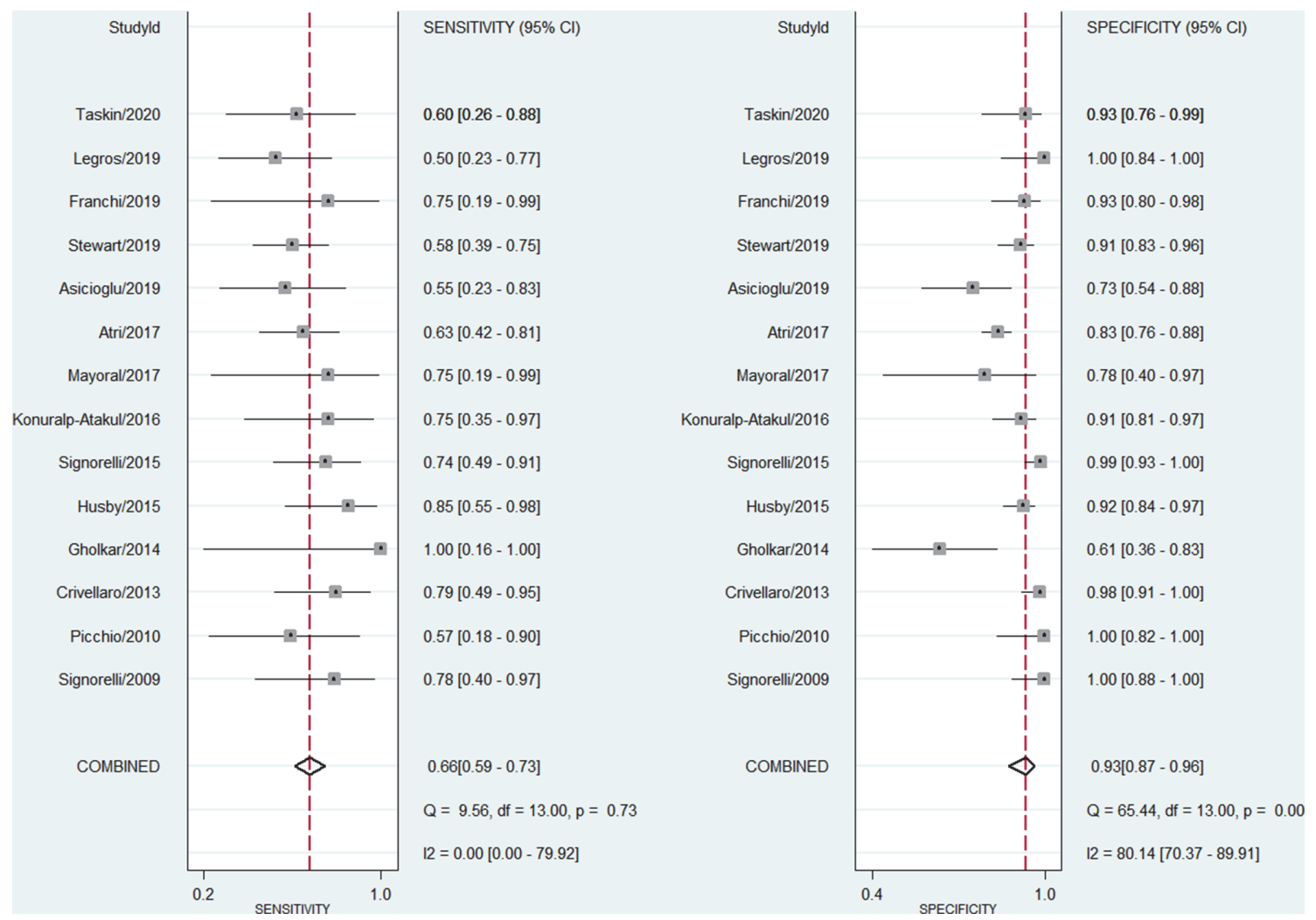

\section{Abstract 612 Figure 1}

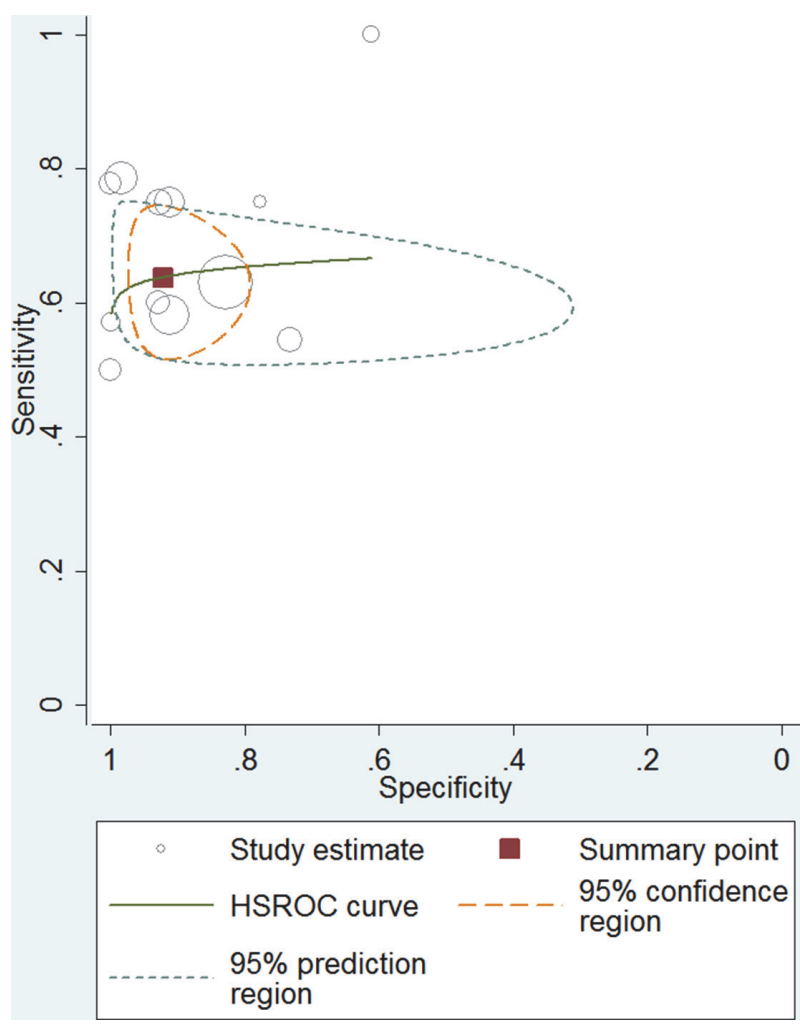

\section{Abstract 612 Figure 2}

node metastases in high-risk endometrial cancer were 0.66 (95\% CI: $0.59-0.73), 0.93 \quad(95 \%$ CI: $0.87-0.96)$ and 0.72 respectively.
Conclusion* Despite the widespread use of 18F-FDG PET or PET/CT for imaging staging in patients with high-risk endometrial cancer, this study show the moderated sensibility of this technique to diagnostic the lymph node metastasis. Therefore, the actual usefulness of this technique for the diagnosis of lymph node metastases is limited, especially nowadays, with the arrival and implementation of the sentinel node.

\section{$615 \quad$ ABSTRACT WITHDRAWN}

\section{VALIDATION OF MODEPLEX TECHNOLOGY FOR THE DETERMINATION OF POLE MUTATIONS IN ENDOMETRIAL CARCINOMA SAMPLES}

${ }^{1}$ E Dorca Duch*, ${ }^{2} \mathrm{~A}$ Velasco, ${ }^{2} \mathrm{D}$ Cuevas, ${ }^{2} \mathrm{M}$ Vaquero, ${ }^{1} \mathrm{~A}$ Rodriguez, ${ }^{1} \mathrm{E}$ Guerra, ${ }^{1} \mathrm{~A}$ Vidal, 1;2X Matías-Guiu Guía. 'Bellvitge Universitary Hospital, Pathology, L'Hospitalet de Llobregat, Spain; ${ }^{2}$ Arnau de Vilanova Universitary Hospital , Pathology, Lleida, Spain

\subsection{6/ijgc-2021-ESG0.175}

Introduction/Background* The current molecular classification of endometrial cancer recognizes 4 subtypes with prognostic and therapeutic implications: 1) Copy-Number-High, 2) Hypermutated, 3) Ultramutated (POLE-mutated) and 4) CopyNumber-Low.

To extrapolate this classification into clinical practice, some authors have proposed a simplified scheme using three immunohistochemical markers and a molecular test (POLE mutation analysis). The mutational determination can be carried out 